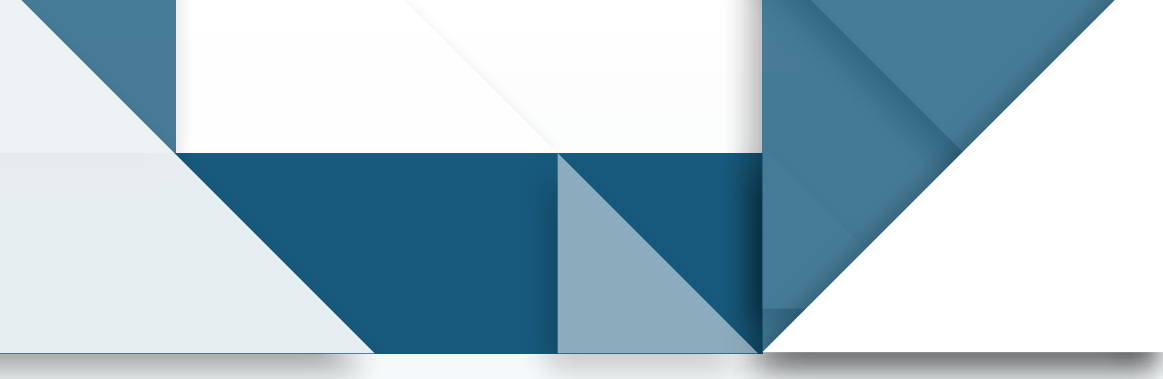

\title{
1) Influencia curricular en el desempeño en el área de matemáticas de las pruebas PISA (2012)
}

- Curricular Influence on Math Performance in the PISA Tests (2012)

- Influência curricular no desempenho na área de matemáticas das provas PISA (2012)

\section{Alexánder Orobio-Montaño* Pedro Nel Zapata-Castañeda **}

\section{Resumen}

El presente artículo muestra los resultados de un estudio cualitativo de casos llevado a cabo en cuatro instituciones educativas de la Ciudad de Bogotá entre los años 2012 y 2015. La investigación tuvo como objetivo principal determinar la influencia de los currículos prescrito y aplicado en el desempeño de los estudiantes en la prueba PISA (2012) en el área de matemáticas. El currículo prescrito se caracterizó a través de una revisión documental de los planes de estudio en cada institución y el currículo aplicado se caracterizó a través de entrevistas no estructuradas a un grupo de profesores, algunos de los cuales posibilitaron la observación directa de sus prácticas docentes. De igual forma, se determinaron los criterios de desempeño de la prueba PISA (2012) por medio de la revisión documental del marco conceptual de esta prueba. Los resultados revelaron que existen apreciables diferencias entre lo que se propone en los currículos prescritos, lo que se enseña por parte de los profesores a través del currículo aplicado y los criterios de desempeño en la prueba PISA, lo que explica los bajos resultados de los estudiantes en el área de matemáticas en esta prueba internacional. Adicionalmente, se evidenció que el pensamiento del profesor de matemáticas influye en el desarrollo de las competencias pretendidas en esta área y la necesidad de mejorar la formación en didáctica de las matemáticas del profesorado.

Palabras clave

Currículo; criterios de desempeño; competencias matemáticas; PISA

\section{Abstract}

This paper discloses the results of a qualitative case study conducted in four schools in Bogotá between the years 2012 and 2015. The main goal of the investigation was to determine the influence of the prescribed and applied curricula on the math performance of students in the PISA test (2012). The prescribed curriculum was characterized through a documentary review of the curriculum of each school, and the applied curriculum was characterized through non-structured interviews with a group of teachers, some of who made

Doctor en Educación y Sociedad, Docente investigador, Universitaria Agustiniana "Uniagustiniana", Facultad de Ciencias Económicas y Administrativas, Docente de matemáticas y física Secretaría de Educación de Bogotá, Colombia. Correo electronico: alexander.orobiom@uniagustiniana. edu.co

orcid.org/0000-0003-4491-4835

** Doctor en Educación, docente investigador, Universidad de La Salle, Facultad de Ciencias de la Educación, Bogotá, Colombia.

Correo electrónico: pnzapata@unisalle.edu.co orcid.org/0000-0001-7042-8983 
it possible to directly observe their teaching practices. The performance criteria of the PISA test (2012) were also determined through documentary review of the conceptual framework of the test. The results revealed significant differences between what prescribed curricula propose, what teachers teach through the applied curriculum, and the performance criteria in the PISA test, which explains the students' low math scores in the international test. In addition, it was shown that the math teacher's thinking influences the development of the intended competences in this area and the need to improve the teachers' training in teaching math.

Keywords

Curriculum; performance criteria; mathematical competence; PISA

\section{Resumo}

presente artigo apresenta os resultados de um estudo qualitativo de casos realizado em quatro instituições educativas da cidade de Bogotá entre os anos 2012 e 2015 . A pesquisa teve como objetivo principal determinar a influência dos currículos prescrito e aplicado no desempenho dos estudantes na prova PISA (2012) na área de matemáticas. $\bigcirc$ currículo prescrito caracterizou-se através de uma revisão documental dos planos de estudo de cada instituição e o currículo aplicado caracterizou-se através de entrevistas não estruturadas a um grupo de professores, que possibilitaram a observação direta das suas práticas docentes. Igualmente, determinaram-se os critérios de desempenho da prova PISA (2012) por meio da revisão documental do marco conceitual desta prova. Os resultados revelaram que existem apreciáveis diferenças entre as propostas dos currículos prescritos, os ensinos dos professores através do currículo aplicado e os critérios de desempenho da prova PISA. Isso explica os baixos resultados dos estudantes na área de matemáticas nesta prova internacional. Adicionalmente, evidenciou-se que o pensamento do professor de matemáticas influi no desenvolvimento das competências previstas nesta área e a necessidade de melhorar a formação em didática das matemáticas do professorado.

Palavras-chave

Currículo; critérios de desempenho; competências matemáticas; PISA 


\section{Introducción}

En una época en la cual se encuentra vigente el debate sobre la calidad de la educación, la discusión sobre la eficacia y la eficiencia de los sistemas educativos ha tomado relevancia para agencias como el Banco Mundial y la Unesco, entre otras, que sin duda influenciaron la política pública educativa en Colombia, desde la concepción de educación que se expresó en el artículo 67 de la Constitución Política de 1991. Sin ir más lejos, en el escenario nacional e internacional la evaluación de los aprendizajes de los estudiantes a través de pruebas estandarizadas se ha establecido como prioridad para medir la calidad de educación que reciben los jóvenes en los centros educativos.

En el caso colombiano, los resultados de la prueba PISA 2012 mostraron que de los 9073 estudiantes del país que participaron, cerca del $74 \%$ no alcanzó el desempeño mínimo esperado, es decir, se clasificó por debajo del nivel básico, lo que puede ser considerado, sin vacilación, como un fracaso colectivo desde el punto de vista académico y de la calidad del sistema educativo colombiano. Todo esto demanda un seguimiento más riguroso a los desempeños de los estudiantes en las próximas participaciones en las pruebas PISA.

En este contexto, se presentan los resultados de una investigación doctoral llevada a cabo en la línea de investigación sobre "Políticas públicas, calidad de la educación y territorio", del Doctorado en Educación y Sociedad de la Universidad de La Salle, que tuvo como objetivo determinar la influencia de los currículos prescrito y aplicado de matemáticas en los desempeños de los estudiantes de cuatro colegios que participaron en la prueba PISA (2012) en esta área. La investigación se llevó a cabo a través de un estudio de caso, con el propósito de comprender e interpretar la particularidad del fenómeno de forma significativa en cada institución participante.

\section{Antecedentes}

La evaluación internacional de alumnos a través de la prueba PISA y la medición de las actitudes e implicaciones de los alumnos hacia y en las ciencias, ha sido abordada en diferentes países desde varias perspectivas y contextos.

En Colombia, el Instituto Colombiano para la Evaluación de la Educación (Icfes) (2012) señaló que el país participó por primera vez en las pruebas PISA, en 2006, año en el que hubo 57 países; mientras que en 2009 participaron 67 países. Los resultados de estas evaluaciones ofrecieron un perfil de los desempeños de los estudiantes además de información acerca de su contexto personal, familiar y escolar. A partir de la experiencia acumulada en PISA 2006 y 2009, el Icfes continuó a cargo de la participación de Colombia en el nuevo ciclo PISA 2012, el cual llevó a cabo su fase piloto en 2011.

Por otra parte, López, Insignares y Rodríguez (2011) exponen un panorama sobre las investigaciones relacionadas con las estrategias de aprendizaje en estudiantes de educación media en el ámbito nacional e internacional. Las fuentes consultadas coinciden en clasificar las estrategias de aprendizaje en diferentes tipos, de manera que unas se encauzan por ayudar al estudiante a codificar y retener la información, mientras otras le permiten elaborar, interpretar e incluso utilizar de manera oportuna y eficaz los conocimientos adquiridos.

Así mismo, Jola (2011) llevó a cabo una investigación en la cual se buscó evidencia de los determinantes de la calidad de la educación en Colombia a través del estudio de los resultados de la prueba PISA 2006. Mediante un análisis econométrico encontró que los recursos físicos del plantel, el currículo o contenido 
académico y la cualificación de los profesores son determinantes positivos en los resultados de la prueba.

El estudio realizado por Barrera, Maldonado y Rodríguez (2012) es una propuesta de política educativa para Colombia que permite mejorar la calidad de la educación básica, secundaria y media, y así convertirla en una herramienta efectiva de movilidad social. Se muestra que Colombia ha tenido avances importantes en cobertura, sin embargo, las mejoras en términos de calidad han sido marginales e inequitativas.

En el campo internacional, la OCDE (2011) patrocinó un estudio sobre la relación entre la autonomía de los centros escolares y el rendimiento en las pruebas PISA. El estudio mostró que en los países donde los centros educativos tienen una mayor autonomía respecto a los temas de enseñanza y a la forma como se evalúa a los estudiantes, tienden a un mejor rendimiento.

El trabajo desarrollado por Inostroza (2014) tuvo como objetivo principal realizar un ejercicio analítico y reflexivo en torno al fracaso en la matemática escolar, cuestionando aquellos enfoques en los cuales se legitiman discursos que atribuyen directamente al estudiante un presunto déficit que sería la causa de su fracaso. Más bien, se propone un direccionamiento en la reflexión hacia las formas de razonar y de enseñar la matemática, con miras a buscar respuestas sobre el fracaso en matemática, en torno a aquellas áreas poco exploradas y que potencialmente podrían mostrar luces respecto a la problemática provocada por las formas tradicionales de razonar y enseñar la matemática escolar.

La investigación realizada por Tiramonti (2014) es un análisis sobre las reformas educativas implementadas en América Latina en la década de 1990 que introdujeron cambios en los modos de regulación de los sistemas educativos basados en la adopción de mecanismos de evaluación de los logros de los alumnos.

Ahora bien, los estudios abordados dan cuenta de los intereses investigativos de instituciones, gobiernos y académicos frente a las pruebas PISA. Aspectos como la convivencia escolar, los ambientes socioeconómicos de los estudiantes, la razón social de las escuelas, la incidencia de género de los estudiantes en el rendimiento escolar, la educación infantil de los niños y niñas, entre otros, han sido indagados. Todos estos estudios pretenden explicar el rendimiento escolar de los estudiantes en PISA y, por supuesto, abren la discusión para la búsqueda de mejores resultados.

\section{Marco teórico}

\section{El contexto de la prueba PISA 2012}

La Organización para la Cooperación y el Desarrollo Económico (OCDE) constituye un foro único en su género, que se distingue por el trabajo conjunto de los gobiernos de treinta países democráticos, inicialmente para afrontar los retos económicos, sociales y medioambientales que plantea la globalización. La OCDE está 
a la vanguardia de los esfuerzos emprendidos para entender los cambios y preocupaciones del mundo actual, como la gobernanza, la economía de la información y los desafíos que plantea el envejecimiento de la población, con el fin de ayudar a los gobiernos a responder a dichos cambios.

Por consiguiente, para responder a la necesidad de disponer de datos sobre el rendimiento escolar que sean comparables internacionalmente, la OCDE puso en marcha en 1997 el Programa para la Evaluación Internacional de Alumnos (PISA, por su sigla en inglés), el cual representa el compromiso de los gobiernos miembros de examinar, de forma periódica y en un marco común internacional, los resultados de los sistemas de educación en función de los logros alcanzados por los alumnos (OCDE, 2008). PISA es actualmente el programa internacional más exhaustivo y riguroso para evaluar el rendimiento académico y para recabar información acerca de los factores estudiantiles, familiares e institucionales que pueden ayudar a explicar las diferencias de rendimiento (Caraballo, Rico y Lupiañez, 2013).

\section{La enseñanza de las matemáticas desde la perspectiva del Ministerio de Educación Nacional de Colombia (MEN)}

La matemática como ciencia es una actividad en la cultura y en la historia y, por tanto, condicionada por ellas, en la cual se utilizan distintos recursos lingüísticos y expresivos para plantear y solucionar problemas tanto internos como externos a las matemáticas mismas (MEN, 2003). En la búsqueda de su enseñanza, la educación matemática implica una actividad intelectual intensa de carácter explicativo, que se sostiene sobre el aprecio por la belleza formal, las nociones de prueba y argumentación, y que se expresa mediante una gran variedad de acciones, términos, símbolos, técnicas, actitudes y recursos. En la educación matemática como disciplina científica, la didáctica de las matemáticas tiene como objeto delimitar y estudiar los problemas que surgen durante los procesos de organización, comunicación, transmisión, construcción y valoración del conocimiento matemático (D'Amore, 2006).

Los cinco procesos generales de las matemáticas desde las perspectivas del MEN

Como criterios generales en la enseñanza de las matemáticas se contemplan cinco procesos desde los Lineamientos Curriculares de Matemáticas, como son: a) formular y resolver problemas; b) modelar procesos y fenómenos de la realidad; c) comunicar; d) razonar, formular y comparar, y e) ejercitar procedimientos y algoritmos (MEN, 1998).

\section{Formular y resolver problemas}

Este es un proceso presente a lo largo de todas las actividades curriculares de las matemáticas y no una actividad aislada y esporádica. Más aun, podría convertirse en el principal eje organizador del currículo de matemáticas, porque las situaciones problema proporcionan el contexto inmediato donde el quehacer matemático cobra sentido, en la medida en que las situaciones que se aborden estén ligadas a experiencias cotidianas y, por ende, sean más significativas para los alumnos.

\section{Modelar procesos y fenómenos de la realidad}

Un modelo puede entenderse como un sistema figurativo mental, gráfico o tridimensional que reproduce o representa la realidad en forma esquemática para hacerla más comprensible. Es una construcción o artefacto material o mental, 
un sistema $O$, a veces, se dice también, una estructura, que puede usarse como referencia para lo que se trata de comprender; una imagen analógica que permite volver cercana y concreta una idea o un concepto para su apropiación y manejo.

\section{Comunicar}

A pesar de que suele repetirse lo contrario, las matemáticas no son un lenguaje, pero ellas pueden construirse, refinarse y comunicarse a través de diferentes lenguajes con los que se expresan y representan, se leen y se escriben, se hablan y se escuchan. La adquisición y dominio de los lenguajes propios de las matemáticas han de ser un proceso deliberado y cuidadoso que posibilite y fomente la discusión frecuente y explícita sobre situaciones, sentidos, conceptos y simbolizaciones, para tomar conciencia de las conexiones entre ellos y propiciar el trabajo colectivo, en el que los estudiantes compartan el significado de las palabras.

\section{Razonar, formular y comparar}

El desarrollo del razonamiento lógico comienza en los primeros grados, apoyado en los contextos y materiales físicos que permiten percibir regularidades y relaciones, hacer predicciones y conjeturas, justificar o refutar esas conjeturas, dar explicaciones coherentes, proponer interpretaciones y respuestas posibles, y adoptarlas o rechazarlas con argumentos y razones.

\section{Ejercitar procedimientos y algoritmos}

Este proceso implica comprometer a los estudiantes en la construcción y ejecución segura y rápida de procedimientos mecánicos o de rutina, también llamados algoritmos, procurando que la práctica necesaria para aumentar la velocidad y precisión de su ejecución no oscurezca la comprensión de su carácter de herramientas eficaces y útiles en unas situaciones $u$ otras, y que, por tanto, pueden modificarse, ampliarse y adecuarse a situaciones nuevas o aun hacerse obsoletas y ser sustituidas por otras.

\section{Concepto de currículo prescrito y currículo aplicado asumidos en la investigación}

En esta investigación se entendió como currículo prescrito el conjunto de acciones y pretensiones de la institución educativa para la enseñanza de las matemáticas, representadas en los planes de estudios desde el Proyecto Educativo Institucional (PEI) que caracteriza la cultura educativa.

El currículo prescrito representa entonces, la filosofía institucional, es decir, la misión y visión en educación matemática que orienta la formación de los estudiantes. Adicionalmente, este currículo da cuenta de la interpretación que hace la institución de las políticas formuladas por los organismos estatales en materia de gestión curricular y evaluación de la calidad de la educación. 
Por otro lado, se asumió como currículo aplicado el conjunto de prácticas educativas y de enseñanza de las matemáticas que llevan a cabo los profesores en las que se usan diversos recursos lingüísticos y expresivos para el planteamiento y solución de problemas internos o externos a las matemáticas. El currículo aplicado contempla la enseñanza de las matemáticas como una disciplina que supone una intensa actividad intelectual de carácter explicativo, basada en la valoración de la belleza formal, las nociones de prueba y argumentación, y expresada gracias a una inmensa diversidad de acciones, términos, símbolos, técnicas, actitudes y recursos.

\section{Metodología}

Bajo las perspectivas del paradigma cualitativo (Vasilachis, 2006), y en el contexto cultural de las prácticas educativas de los profesores de matemáticas, se estableció la relación entre los currículos prescrito y aplicado de matemáticas, con los desempeños de los estudiantes de cuatro colegios en el área de matemáticas de las pruebas PISA 2012. De hecho, la relación entre los currículos y los desempeños se determinaron en tres aspectos fundamentales: a) la caracterización de los currículos a través de la inmersión en la vida cotidiana y la realización de entrevistas aplicadas a los informantes y la observación de las prácticas docentes; b) la valoración y el intento por descubrir la perspectivas de los informantes sobre sus propias prácticas en la enseñanza de las matemáticas, y c) la consideración de la investigación como un proceso interactivo entre el investigador y los informantes, de carácter descriptiva, analítica y explicativa que privilegió la caracterización de los currículos prescrito y aplicado, y la influencia de estos en los desempeños de los estudiantes en las pruebas PISA (2012) en el área de matemáticas.

\section{Método de investigación}

La complejidad de cada institución educativa fue abordada desde el método de estudio de casos (Stake, 2005). La intención principal fue analizar en detalle la correspondencia de los currículos prescrito y aplicado con los criterios de desempeños de la prueba PISA (2012) en el área de matemáticas, examinando la particularidad, complejidad y singularidad de dichos currículos para lograr comprender su influencia en los desempeños de los estudiantes en la prueba internacional.

\section{Colegios e informantes seleccionados}

Para el desarrollo de la investigación se seleccionaron cuatro instituciones educativas de 48 pertenecientes a la Secretaría de Educación de Bogotá, que participaron en la prueba PISA (2012), 40 de ellas caracterizadas como oficiales, y 8 , de educación contratada o de convenio. El acercamiento a estas se realizó a través de una invitación formal dirigida al representante legal de la institución y la socialización del proyecto de investigación con los directivos y los docentes de matemáticas. Esta experiencia permitió seleccionar una institución en cada una de las localidades de Bosa, Kennedy, Fontibón y Ciudad Bolívar. Para llevar a cabo el análisis del currículo prescrito se obtuvieron los planes de estudios del área de matemáticas en físico y digital de las cuatro instituciones educativas participantes. Adicionalmente, para caracterizar el currículo aplicado, se conformó un grupo de 17 profesores con los cuales se llevaron a cabo entrevistas no estructuradas en profundidad. De este grupo, se seleccionaron 6 docentes para las observaciones de las prácticas docentes: 2 por colegio, exceptuando la institución educativa 4 en la que ninguno de los informantes autorizó la observación de la práctica docente. 


\section{Análisis de datos y resultados}

El análisis de la información recolectada se llevó a cabo a partir de los lineamientos y procedimientos señalados por Fernández (2006). A continuación, se presentan los resultados obtenidos, considerados como reflexiones derivadas de los hallazgos en cada una de las instituciones estudiadas.

\section{Criterios de desempeños en las pruebas PISA 2012 en el área de matemáticas}

El análisis documental del marco conceptual de la prueba PISA (2012) en el área de matemáticas mostró que esta se encuentra estructurada en dos dimensiones, cada una conformada por tres categorías, a saber: alfabetización matemática (contenido matemático, procesos matemáticos y contextos matemáticos) y solución de problemas (contexto del problema, naturaleza del problema y procesos de resolución de problemas). A continuación se describen los principales criterios que determinan las tres categorías de esta prueba.

\section{El contenido matemático}

La comprensión del contenido matemático y la habilidad para aplicar ese conocimiento a la solución de problemas contextualizados son importantes para los ciudadanos en el mundo de hoy. Los criterios para su evaluación son:

- Cambio y relaciones: los tipos fundamentales de cambio y el reconocimiento de cuándo ocurren para así utilizar modelos matemáticos adecuados y describir y predecir el cambio.

- Espacio y forma: abarca una gama amplia de fenómenos que se encuentran en todas partes en nuestro mundo visual: patrones, propiedades de los objetos, posiciones y orientaciones, representaciones de objetos, decodificación y codificación de la información visual, navegación e interacción dinámica con formas reales y con sus representaciones.

- Cantidad: puede ser el aspecto matemático más dominante y esencial al interactuar y funcionar en nuestro mundo.

- Incertidumbre: esta categoría de contenido incluye el reconocimiento del lugar de la variación en los procesos, con un sentido de la cuantificación y explicación de la variación, reconociendo la incertidumbre y el error en la medición, y el conocimiento de la casualidad.

En cuanto a esta categoría, los resultados del estudio permitieron evidenciar que las cuatro instituciones analizadas enfatizaron en el contenido matemático a través del currículo prescrito y aplicado, las cuales equivalen a los cinco pensamientos matemáticos ya descritos anteriormente. Esto significa que los currículos dan cuenta en forma importante de los conocimientos matemáticos que consideró la evaluación PISA (2012). 


\section{Los procesos matemáticos}

Formular situaciones matemáticamente hace referencia a la capacidad de las personas de reconocer e identificar oportunidades para utilizar las matemáticas, esto es, traducir un problema en un contexto natural a una forma matemática. Incluye actividades como:

- Identificar los aspectos matemáticos de un problema situado en un contexto del mundo real e identificar las variables significativas.

- Identificar las restricciones y suposiciones detrás de cualquier modelo matemático y las simplificaciones deducidas del contexto.

- Usar la tecnología (por ejemplo, las hojas de cálculo o la lista de herramientas en una calculadora graficadora) para presentar la relación matemática inherente en un problema contextualizado.

- Emplear conceptos, hechos, procedimientos y raciocinio matemático.

- Diseñar e implementar estrategias para encontrar soluciones matemáticas.

- Aplicar reglas matemáticas, algoritmos y estructuras cuando se buscan soluciones.

- Manipular números, datos e información estadística y gráfica, expresiones algebraicas y ecuaciones y representaciones geométricas.

- Interpretar, aplicar y evaluar los resultados matemáticos.

- Evaluar la racionalidad de la solución matemática en el contexto de un problema del mundo real.

- Entender cómo el mundo real tiene efecto en los resultados y cálculos de un procedimiento o modelo matemático, para emitir juicios contextuales sobre cómo los resultados deben ajustarse o aplicarse.

Si bien, los resultados del estudio demostraron que las cuatro instituciones analizadas enfatizaron en unos pocos aspectos de esta categoría a través del currículo prescrito y tan solo 8 de los 17 profesores de matemáticas los hicieron a través del currículo aplicado. Existe una falta de correspondencia entre los currículos y los criterios de desempeños de la evaluación PISA (2012), pues los currículos además de enfatizar de manera aceptable se centraron en los procesos de enseñanza, es decir en el docente, contrario a los criterios de PISA que se centraron en los aprendizajes de los estudiantes. Dicho de otra forma, en esta categoría los estudiantes deben demostrar el aprendizaje de los conocimientos matemáticos y el desarrollo de las competencias matemáticas desde las perspectivas de PISA.

\section{Los contextos del problema}

Los contextos se clasifican en cuatro categorías (personal, ocupacional, social y científico):

- Personal: los problemas se ubican en contextos personales, es decir, en actividades propias del estudiante, de la familia o de un grupo de compañeros.

- Ocupacional: los problemas que se presentan en un contexto ocupacional se centran en el mundo del trabajo.

- Social: los problemas se ubican en contextos sociales de la comunidad.

- Científico: los problemas que se presentan en contextos científicos relacionan la aplicación de las matemáticas en el mundo natural, y los problemas y temas relacionados con la ciencia y la tecnología. 
Habría que agregar, en cuanto a esta categoría, que los resultados del estudio permitieron evidenciar que 3 de las 4 instituciones analizadas enfatizaron en ella a través del currículo prescrito, y tan solo 8 de los 17 profesores de matemáticas entrevistados los hicieron a través del currículo aplicado, lo que permite explicar una discreta relación de los currículos con los desempeños de los estudiantes en la prueba del área de matemáticas de PISA (2012).

En cuanto a la prueba de solución de problemas, esta constituye una base para el aprendizaje futuro, para la participación productiva en la sociedad y para la realización de las actividades personales.

A continuación, se presentan algunos hallazgos sobre los currículos prescrito y aplicado a partir de las categorías propuestas por PISA en la solución de problemas, a saber: el contexto del problema, la naturaleza de la situación problema y los procesos de resolución de problemas.

\section{Contexto del problema}

La familiaridad y la comprensión que tenga una persona con el contexto de un problema afectarán la manera como lo resuelva. Por esta razón, se han identificado dos dimensiones para asegurar que las tareas se inscriben en un rango de contextos que resulten auténticos y de interés para los estudiantes de 15 años: el ambiente (tecnológico o no) y el foco (personal o social). En efecto, los resultados del estudio permitieron evidenciar que 3 de las 4 instituciones analizadas acentuaron en esta categoría de forma aceptable a través del currículo prescrito, así mismo lo hicieron 8 de los 17 profesores de matemáticas entrevistados a través del currículo aplicado, lo que permite explicar los bajos aciertos de los estudiantes en la prueba PISA (2012).

\section{Naturaleza de la situación problema}

Las situaciones problema pueden ser interactivas o estáticas. Las primeras, con frecuencia, surgen cuando se encuentran, en principio, artefactos en el mundo real como las máquinas expendedoras de boletos, sistemas de aire acondicionado o teléfonos celulares, especialmente si las instrucciones de uso de tales dispositivos no son claras o no están disponibles. Entender cómo controlar tales dispositivos es un problema que se puede enfrentar en la vida diaria. En estas situaciones, suele ocurrir que una parte de la información relevante no está disponible desde el principio. Por ejemplo, es posible que el efecto de aplicar una operación (por ejemplo, oprimir un botón en un teléfono celular) no se conozca o no se pueda deducir, sino que deba inferir mediante una interacción con el escenario a través del cual se desarrolla la operación (oprimir el botón) y elaborar una hipótesis sobre su funcionamiento basado en el resultado. En general, debe llevarse a cabo algo de exploración o experimentación para adquirir el conocimiento necesario para controlar el dispositivo. Otro escenario común aparece cuando una persona tiene 
que averiguar cuál es el problema o cuando hay una falla o la máquina no funciona adecuadamente. En este caso, hay una cierta cantidad de experimentación necesaria para recopilar los datos de las circunstancias en las cuales un objeto tecnológico no funciona.

En las situaciones problema estáticas, por su parte, toda la información relevante está disponible desde el principio y la situación no cambia en el curso de resolver el problema. Un ejemplo de lo anterior es la escogencia del analgésico correcto, dados unos detalles suficientes sobre el paciente, los síntomas que presenta y los analgésicos disponibles. Aquí se cuenta con un número muy definido de alternativas y de restricciones para tomar la decisión que satisfaga todos los requerimientos (González, Sánz, Leguizamón y Juyar, 2012). Cabe resaltar que en esta categoría, los resultados del estudio permitieron evidenciar que ninguna de las cuatro instituciones analizadas enfatizó en ella a través del currículo prescrito y tan solo 4 de los 17 profesores de matemáticas los hicieron a través del currículo aplicado, lo que permite manifestar los prudentes desempeños de los estudiantes en la prueba de resolución de problemas en la evaluación PISA (2012).

\section{Procesos de resolución de problemas}

La resolución de problemas implica una diversidad de procesos y comprensión de situaciones de la cotidianidad, de otras ciencias y propias de las matemáticas las cuales se describen a continuación:

- Explorar la situación problema: observarla, interactuar con ella, investigar la información, buscar limitaciones u obstáculos

- Comprender la información dada y la información descubierta, al tiempo que se interactúa con la situación problema y se demuestra comprensión de los conceptos relevantes

- Representación y formulación: el objetivo es construir una representación mental coherente de la situación problema, es decir, un modelo para la situación o un modelo para el problema. Para hacerlo, hay que seleccionar la información relevante, organizarla e integrarla con el conocimiento relevante anterior. Esto implica:

- Planeación, que consiste en establecer las metas, incluida la clarificación de la meta global y el establecimiento de submetas, cuando sea necesario, y el diseño de un plan o estrategia para alcanzar la meta establecida, incluidos los pasos por seguir

- La ejecución, que consiste en llevar a cabo el plan.

- Reflexión: consiste en revisar el avance hacia la consecución de la meta en cada etapa, incluida la verificación de los resultados finales e intermedios, detectar eventos inesperados y tomar acciones correctivas cuando sea necesario.

- Reflexionar sobre las soluciones desde diferentes perspectivas, evaluar críticamente las soluciones y suposiciones alternativas, y buscar la información adicional que se requiera (González, Sánz, Leguizamón y Juyar, 2012)

Los resultados permitieron demostrar que ninguna de las cuatro instituciones analizadas enfatizaron en los procesos de resolución de problemas a través del currículo prescrito, así mismo que solo 4 de los 17 profesores de matemáticas entrevistados, incluidos los 6 que permitieron las observaciones de sus prácticas docentes, consideraron esta categoría, lo que 
permite manifestar además los bajos desempeños de los estudiantes en la prueba PISA (2012), el fracaso académico como realmente ocurrió.

\section{Currículo prescrito}

En cuanto al currículo prescrito, se hizo una revisión documental de los planes de estudio de las cuatro instituciones educativas. Esta tarea fue orientada por las categorías de la prueba de alfabetización matemática (contenido matemático, procesos matemáticos, contextos matemáticos) y la prueba de resolución de problemas (contexto del problema, naturaleza de la resolución de problemas y procesos de resolución de problemas).

\section{Institución educativa: código 1.7}

A partir de las categorías: contenido matemático, procesos matemáticos, contextos matemáticos, naturaleza de la resolución de problemas y procesos de resolución de problemas, se identificó que, en la de contenido matemático, el plan de estudio contempla en su totalidad los objetos matemáticos de enseñanza/aprendizaje desde las políticas en educación matemática. En cuanto a los procesos matemáticos, se reconoció una fortaleza en los tres aspectos fundamentales: formular situaciones matemáticamente, emplear conceptos, hechos y procedimientos, y raciocinio matemático. Por último, el currículo prescrito no enfatiza en las cuatro categorías restantes.

\section{Institución educativa: código 2.8}

En la caracterización del currículo prescrito, se identificó que la concepción de educación matemática se haya orientada por los estándares básicos de competencias en matemáticas, pues el documento está estructurado en dicho enfoque.

En este sentido, desde las seis categorías que permitieron caracterizar el plan de estudios se advirtió que, en la categoría contenido matemático, el plan de estudio solo contempla 3 de 5 objetos matemáticos del proceso enseñanza/aprendizaje. Así mismo, en la categoría procesos matemáticos se identificó una debilidad en cuanto a los 5 procesos generales, ya que el documento no presenta una estructura fundamentada para la enseñanza de los conocimientos matemáticos, centrada en el estudiante como lo propuso la prueba PISA (2012). Por último, el documento no da cuenta de las cuatro categorías restantes.

\section{Institución educativa: código 3.9}

En esta institución se evidenció que en la categoría contenido matemático, el plan de estudios contempla los cinco objetos matemáticos del proceso enseñanza/ aprendizaje; en la categoría procesos matemáticos, se identificó una fortaleza en cuanto a los cinco procesos generales, pues el documento presenta una estructura fundamentada para la enseñanza de los conocimientos matemáticos centrada en 
el rol del docente como mediador en el aprendizaje del estudiante, contrario a los criterios de PISA (2012) que sugieren que el estudiante sea quien se apropie de dichos procesos.

En cuanto al contexto del problema, se encontró escasa información relacionada con los contextos, es decir, con los diferentes escenarios de la vida personal y científica donde se pueden aplicar los conocimientos matemáticos a través del enfoque de resolución de problemas. El plan de estudios no dio cuenta de las tres categorías restantes.

\section{Institución educativa: código 4.19}

Desde las categorías mencionadas, se hicieron los siguientes hallazgos: el contenido matemático contempló los cinco objetos matemáticos del proceso de enseñanza/aprendizaje desde las políticas en educación matemática; en los procesos matemáticos, muy similar a la institución educativa código 3.9, se identificó una fortaleza en cuanto a los cinco procesos generales, pues el documento presenta una estructura fundamentada para la enseñanza de los conocimientos matemáticos centrada en el rol del docente como mediador en el aprendizaje del estudiante. Por último, el currículo prescrito no dio cuenta en absoluto de las cuatro categorías restantes.

\section{Currículo aplicado}

Como se mencionó anteriormente, la caracterización del currículo aplicado se llevó a cabo mediante una entrevista no estructurada. Cabe resaltar que esta fue considerada un instrumento pertinente para obtener información dado que, en la jornada de socialización y acercamiento a las instituciones y a los 17 profesores participantes, se identificó que ninguno conocía las pruebas PISA 2012, a pesar de que manifestaron haber escuchado por algunos medios de comunicación información parcial sobre los resultados obtenidos en dicha evaluación internacional.

\section{Institución educativa código 1.7}

Los hallazgos mostraron que de las 6 categorías encontradas en la prueba PISA, en 2 de ellas se enfatizan con mayor rigor los criterios propios de la enseñanza/aprendizaje de las matemáticas: las de contenidos matemáticos y procesos matemáticos. Por consiguiente, los procesos matemáticos son una actividad constante en la práctica docente y el pensamiento matemático de los profesores.

Cabe resaltar que, si bien se enfatiza en los procesos de la resolución de problemas, propios de la categoría los contextos, el currículo aplicado no da cuenta de ello desde la praxis del docente, es decir, se enfatiza muy poco o nada, lo cual se evidencia de la misma manera en las categorías naturaleza de la situación problema y procesos de resolución de problemas.

\section{Institución educativa código 2.8}

En esta institución se encontró que de las 6 categorías ya expuestas, el currículo aplicado enfatiza en 2 de ellas con mayor rigor: los contenidos matemáticos y los procesos matemáticos. Mientras tanto, las categorías los procesos de resolución de problemas y los contextos se enfatizan poco o nada desde la praxis del docente, lo cual se evidencia de la misma manera con la naturaleza de las situaciones problema y los procesos de resolución de problemas.

En conclusión, el currículo aplicado carece de rigor, sobre todo en el enfoque de resolución de problemas desde los criterios basados en los estándares básicos de competencias.

\section{Institución educativa código 3.9}

En esta institución se encontró que el currículo aplicado enfatiza significativamente en los contenidos matemáticos, los procesos matemáticos y los contextos. También es importante señalar que el currículo aplicado enfatiza en las 
categorías de procesos de resolución de problemas y los contextos, es decir, se enfatiza constantemente en las cinco categorías. Lo contrario se observa con las categorías naturaleza de la situación problema y procesos de resolución de problemas, los cuales no se manifiestan en los procesos de enseñanza y aprendizaje de las matemáticas en esta institución.

\section{Institución educativa código 4.19}

Los hallazgos mostraron que el currículo matemático carece de actividades matemáticas propias del proceso de enseñanza/aprendizaje en las categorías contexto del problema, naturaleza de la situación problema y procesos de resolución de problemas. Esto indica que, en el enfoque de resolución de problemas, el currículo enfatiza poco o nada en actividades matemáticas.

\section{Currículos prescrito y aplicado y desempeños en PISA 2012}

A partir de la determinación de los criterios de desempeños de la prueba PISA 2012 y la caracterización de los currículos prescrito y aplicado en cada una de las instituciones participantes se procedió a establecer su relación, para lo cual se elaboró una matriz a manera de triangulación que permitió identificar diferencias y similitudes entre ellos. A continuación se presentan los principales hallazgos que dan cuenta de la relación mencionada para cada institución educativa.

\section{Institución educativa código 1.7}

No se encontró una relación fuerte o significativa entre los currículos prescrito y aplicado y los criterios de desempeños de las pruebas PISA. Sin embargo, se reconoce una relación significativa en 2 de las 6 categorías mencionadas: contenido matemático y contextos matemáticos.

\section{Institución educativa código 2.8}

No se encontró una relación fuerte o significativa entre los currículos prescrito y aplicado y los criterios de desempeño de las pruebas PISA. No obstante, se reconoce una relación en dos de las seis categorías mencionadas: contenido matemático y los contextos matemáticos.

\section{Institución educativa código 3.9}

Se encontró una relación aceptable entre los currículos prescrito y aplicado y los criterios de desempeños de las pruebas PISA, solo en cuatro de las seis categorías mencionadas: contenido matemático, los contextos matemáticos, los procesos matemáticos y los procesos de resolución de problemas. 


\section{Institución educativa código 4.19}

Se encontró una relación muy débil entre los currículos prescrito y aplicado y los criterios de desempeños de las pruebas PISA. Sin embargo, se reconoce una relación aceptable en una de las 6 categorías consideradas, la de contenido matemático.

\section{Conclusiones}

Es necesario comenzar recalcando que los desempeños discretos de los estudiantes en la prueba PISA (2012) en el área de matemáticas constituyen una problemática constante en las 4 instituciones educativas incluidas en el estudio. Por tanto, dicha problemática debe ser situada e intervenida de manera responsable, según las características específicas de cada cultura educativa. El análisis e interpretación de esta realidad convoca a las instituciones y a los profesores de matemáticas a buscar una transformación de las prácticas de enseñanza y a apropiarse de los recursos didácticos necesarios para ofrecer una mejor educación matemática, que propenda por el mejoramiento de las demandas de evaluaciones externas a las instituciones educativas como PISA.

La presente investigación mostró que los currículos prescrito y aplicado no contemplan ni abordan los aspectos curriculares que podrían garantizar que los estudiantes desarrollen las competencias matemáticas que evalúa PISA. Igualmente, se confirmó que los currículos prescrito y aplicado no dan cuenta de forma importante de 4 de las 6 categorías que evalúa la prueba PISA 2012 en el área de matemáticas, hablamos de los contextos matemáticos, contextos del problema, naturaleza de la situación problema y procesos de resolución de problemas. Se resalta, sin embargo, que los currículos prescrito y aplicado acertaron de manera significativa en la categoría contenido matemático, y aceptable en la categoría procesos matemáticos.
Mediante la caracterización de los currículos prescrito y aplicado, se identificó en las cuatro instituciones una carencia muy significativa en la dimensión de resolución de problemas propios de las matemáticas, de otras ciencias y de la cotidianidad de los estudiantes de 15 años. Aspectos fundamentales para una educación matemática de calidad, como los contextos matemáticos en lo personal, ocupacional, social y científico, los contextos del problema y los procesos de resolución de problemas, no median la cotidianidad de la educación matemática de los docentes, ni son contemplados por ellos en sus prácticas de enseñanza. Desde luego, esto permite pronosticar el fracaso de los estudiantes en la evaluación de alfabetización matemática de la prueba PISA, pues no cuentan con el desarrollo de competencias que, al respecto, enfatiza la evaluación internacional.

En las 4 instituciones también se comprobó que los currículos prescritos, y en especial los planes de estudio, se enfocan principalmente en los estándares básicos de competencias en matemáticas, sin embargo, dejan de lado o ignoran otros criterios que emergen de reflexiones institucionales o investigativas y que, a su vez, son política pública, como los Lineamientos Curriculares de Matemáticas (MEN, 1998) y la Fundamentación Conceptual del Área de Matemáticas (Icfes, 2007), los cuales, sin duda, conforman un conjunto de herramientas didácticas y pedagógicas para el docente que pueden favorecer los procesos de enseñanza/aprendizaje de los estudiantes en el área mencionada.

A esto se añade que el estudio logró confirmar la necesidad de mejorar la formación en didáctica de las matemáticas del profesorado y, además, que el pensamiento del profesor de matemáticas influye en el desarrollo de las competencias pretendidas en esta área. Al respecto, los 17 profesores entrevistados en las 4 instituciones educativas manifestaron no conocer la prueba PISA (2012), ni mucho menos las competencias 
matemáticas que se evaluaron o se evalúan en esta prueba, lo cual, aunado a las carencias ya descritas de los currículos prescrito y aplicado, explican los bajos desempeños de los estudiantes en el área de matemáticas en la prueba PISA (2012).

De acuerdo con lo anterior, la no correspondencia de los currículos prescrito, aplicado y los criterios de desempeños en la prueba PISA (2012), en el área de matemáticas, convoca a las cuatro instituciones y a los profesores del área a implementar planes de mejoramiento que permitan superar tales resultados, no solo en esta prueba internacional sino en otras en las que participa Colombia. Cabe aclarar que PISA no suministra datos sobre los desempeños individuales por estudiantes, pero la homogeneidad de estos en los desempeños en la prueba de 2012 sustenta tal afirmación.

Ahora bien, pese a que aquí se aplicó el método de estudios de casos, mediante el cual se buscó examinar la singularidad de cada colegio seleccionado en torno a la problemática en cuestión, los resultados permiten inferir algunas situaciones que podrían ser abordadas en el resto de las instituciones educativas del país que participaron en la prueba PISA (2012).

\section{Referencias}

Caraballo, R. M., Rico, L. y Lupiañez, J. L. (2013). Cambios conceptuales en el marco teórico competencial de PISA: el caso de las matemáticas. Revista de Curriculum y Formación del Profesorado, 17(2), 225-241.

Barrera, F., Maldonado, D. y Rodríguez, C. (2012). Calidad de la Educación Básica y Media en Colombia. Diagnóstico y propuestas. Serie de documentos de trabajo, 126. Bogotá: Universidad del Rosario. Facultad de Economía.

D’Amore, B. (2006). Didáctica de la matemática. Bogotá: Magisterio.

Fernández, N. L. (2006). ¿̇Cómo analizar datos cualitativos? Butlletí LaRecerca, 7. Barcelona: Instituto de Ciencias de la Educación, Universidad de Barcelona.

González, M., Sánz, C., Leguizamón, R. y Juyar, J. (2012). Programa Internacional de Evaluación de estudiantes PISA 2012. Guía de Orientación Estudio Principal Colombia 2012. Evaluación de Estudiantes PISA 2012. Bogotá: ICFES.

Instituto Colombiano para la Evaluación de la Educación (lcfes) (2007). Fundamentación Conceptual Área de Matemáticas. Bogotá, D.C.

Instituto Colombiano para la Evaluación de la Educación (Icfes) (2012). Evaluaciones Internacionales. Recuperado de http://www.ICFES.gov.co/investigacion/ evaluaciones-internacionales

Inestroza, A. F. (2014). Entre más sumamos, más nos restan. El fracaso en matemática escolar. En busca de respuestas. Revista Educación y humanidades. Santiago de Chile: Universidad de la Frontera. 
Jola, A. F. (201 1). Determinantes de la calidad de la Educación Media de Colombia: Un análisis de los resultados PISA 2006 y del Plan Sectorial. Universidad Sergio Arboleda. Bogotá, Colombia: Fedesarrollo.

López, D., Insignares R.Y. y Rodríguez, A. (201 1). Estrategias de aprendizaje en estudiantes de Educación Media. Revista de la Facultad de Psicología de la Universidad Cooperativa de Colombia, 130-138.

Ministerio de Educación Nacional (MEN). (1998). Lineamientos Curriculares de Matemáticas. Bogotá.

Ministerio de Educación Nacional (MEN). (2003). Estándares básicos de competencias en matemáticas. Bogotá

Organización para la Cooperación y el Desarrollo Económico (OCDE) (2008). Informe PISA 2006. Competencias científicas para el mundo del mañana. Madrid: Santillana.

Organización para la Cooperación y el Desarrollo Económico (OCDE) (2011). Autonomía y medición de cuentas en los centros educativos: ¿̇Están relacionados con el rendimiento de los estudiantes? PISA in Focus, 9. Recuperado de http://www. oecd.org/PISA/PISAproducts/PISAinfocus/49359322.pdf

Stake, R. (2005). Investigación con estudio de casos. Madrid: Morata.

Tiramonti, G. (2014). PISA test in Latin América: results in context. Buenos Aires: Universidad Nacional de la Plata.

Vasilachis, I. (2006). Estrategias de investigación cualitativa. Barcelona: Gedisa.

\section{Para citar este artículo}

Orobio, A. y Zapata, P. (2017). Influencia curricular en el desempeño en el área de matemáticas de las pruebas PISA (2012). Tecné, Episteme y Didaxis, TED, 42, 97-113. 